\title{
Nuevos registros de pulgas de tlacuaches Didelphis virginiana (Kerr 1792) en Querétaro, México
}

\author{
Norma Hernández-Camacho ${ }^{1 *}$, Santiago Vergara-Pineda ${ }^{2}$, \\ Roxana Acosta-Gutiérrez ${ }^{3}$ y Robert $W$. Jones ${ }^{1}$
}

Introduction: The opossum Didelphis virginiana (Kerr 1792) is one of the eight species of marsupials that live in the northern region of America and is widely distributed in Mexico. There is a large literature in North America of studies on this species, including its ectoparasites. But in Mexico studies are scarce, and so we decided to investigate the opossum ectoparasite species richness in a suburban landscape in Querétaro, México.

Methods: During January to May of 2010, we used 12 tomahawk traps to capture opossums. Each animal was immobilized with tiletilamina, and during recumbence we examined the opossums for ectoparasites. Fleas were collected in vials with $70 \%$ alcohol, then dehydrataded in $100 \%$ alcohol, and clarified with lactic acid for further identification.

Results: We found four flea species on the trapped opossums $(n=26), 14$ males, 12 females. The species were Ctenocephalides felis, Euhoplopsyllus glacialis affinnis, Polygenis martinezbaezi and Polygenis sp. Two of these species are considered as new records for this marsupial host in Mexico, although these species have been recorded previously in this species in the United States and in other marsupial species in Mexico.

Discussion: These fleas are new records for the Mexican opossum parasite fauna. This study, although initially didactic, reveals relevant information about the flea species richness on opossums in fragmented habitats in a suburban landscape in Querétaro. Because fleas have potential as disease and parasite vectors, this new information may be helpful in assessing their effects on human and wildlife health.

Key words: Didelphis virginiana, fleas, suburban landscape, Querétaro, México

\section{Resumen}

El tlacuache Didelphis virginiana (Kerr 1792) es una de las ocho especies de marsupiales que habitan en la región norte del continente americano y se encuentra ampliamente distribuida en México. Se han realizado una gran variedad de trabajos sobre esta especie, entre ellos el estudio de los ectoparásitos de estos marsupiales a lo largo de América. En un ejercicio académico sobre la estimación poblacional de D. virginiana en el Campus

${ }^{1}$ Cuerpo Académico de Ecología y Diversidad Faunística. Facultad de Ciencias Naturales. Universidad Autónoma de Querétaro, Santiago de Querétaro 76230, Querétaro, México. E-mail: norma.hernandez@uaq.mx (NHC) y rjones@uaq. $\mathrm{mx}(\mathrm{RWJ})$

${ }^{2}$ Cuerpo Académico de Biología y Aprovechamiento de la Flora y Microorganismos. Facultad de Ciencias Naturales. Universidad Autónoma de Querétaro, Santiago de Querétaro 76230, Querétaro, México. E-mail: vpinedas@yahoo.com. $\mathrm{mx}(\mathrm{SVP})$

${ }^{3}$ Museo de Zoología, Facultad de Ciencias, Universidad Nacional Autónoma de México, Apartado Postal 70-399, Distrito Federal 04510, México. E-mail: roxana_a2003@yahoo.com.mx (RAG)

${ }^{*}$ Corresponding autor. 
Juriquilla de la Universidad Autónoma de Querétaro, se encontraron cuatro especies de pulgas, Ctenocephalides felis, Euhoplopsyllus glacialis affinnis, Polygenis martinezbaezi y Polygenis sp., la presencia de dos de estas especies de pulgas en $D$. virginiana puede ser considerado como un nuevo registro para México, las pulgas que se identifican en este trabajo han sido registradas previamente en estudios realizados sobre otras especies de marsupiales en Estados Unidos y México. Este estudio, si bien inicialmente de carácter didáctico, arrojó información importante sobre la riqueza de especies de pulgas en tlacuaches que habitan ambientes con influencia humana y el efecto potencial de estos organismos para la salud de los seres humanos y de la fauna silvestre en un paisaje suburbano.

Palabras clave: Didelphis virginiana, pulgas, paisaje suburbano, Querétaro, México.

El tlacuache Didelphis virginiana (Kerr 1792) es una de las ocho especies de marsupiales Introducción que habitan en la región norte de América y se encuentra ampliamente distribuida en México (McManus 1974 y Ceballos et al. 1998). Debido a sus características biológicas y ubicuidad, se han realizado una gran variedad de estudios sobre esta especie, entre los que resaltan los registros de la parasitofauna de este marsupial a lo largo de más de 70 años, lo que ha permitido el registro de 38 especies de helmintos endoparásitos (García-Prieto et al. 2012) mientras que la información sobre los ectoparásitos de los didélfidos para el país ha comenzado a incrementarse en los últimos años (Morrone y Gutiérrez 2005; Whitaker y Morales-Malacara 2005; Whitaker et al. 2007 y GuzmánCornejo et al. 2012) tanto en ambientes naturales como en aquellos dentro de las ciudades o que rodean a éstas, como son los paisajes suburbanos, los cuales, en los últimos años han sido reconocidos por su potencial para albergar comunidades biológicas diversas pese a la constante presión del crecimiento demográfico (Forman y Godron 1986 y Davies et al. 2001).

Un ejemplo de este tipo de paisaje suburbano es el que se puede encontrar en el Campus Juriquilla de la Universidad Autónoma de Querétaro (CJUAQ), localizado 13 kilómetros al norte de la Ciudad de Santiago de Querétaro, en la Delegación de Santa Rosa Jáuregui. El proceso de urbanización de la zona se ha acelerado en los últimos cinco años, sin embargo, alrededor de las instalaciones del CJUAQ todavía existen fragmentos relativamente grandes de hábitat original (selva baja caducifolia) y de vegetación secundaria que sirven de hábitat a varias especies de mamíferos. Por observación directa, se ha podido determinar la presencia de Didelphis virginiana, Spilogale putorius, Urocyon cinereoargenteus, Bassariscus astutus, Otospermophilus variegatus, Silvilagus floridanus, Liomys irroratus y Peromyscus spp.; algunas de estas especies se les considera como generalistas ecológicos debido a que pueden sobrevivir en ambientes con un determinado grado de perturbación y presencia humana, como es el caso de U. cinereoargenteus (Harrison 1997; Buskirk 1999; Sunquist y Sunquist 2001; Gehring y Swihart 2003 y Hernández-Camacho y López-González 2009) o D. virginiana (McManus 1974), sin embargo, el crecimiento del campus como consecuencia del incremento de la matrícula estudiantil de las facultades que se ubican en dicho espacio, permitió considerar estas condiciones adecuadas para 
ejemplificar un caso de estudio con fines didácticos, sobre el efecto de las actividades humanas en las poblaciones de mamíferos silvestres, a partir de dicho estudio, se recabaron una serie de datos, entre ellos la riqueza de ectoparásitos de esta especie de marsupial dentro del CJUAQ. Por lo tanto, se consideró explorar este aspecto en el estudio, así que, el objetivo de este trabajo en particular, fue conocer la riqueza de ectoparásitos de Didelphis virginiana en un paisaje suburbano sometido a distintas presiones ecológicas como consecuencia de actividades humanas en el Campus Juriquilla de la Universidad Autónoma de Querétaro.

\section{Material}

El Campus Juriquilla de la Universidad Autónoma de Querétaro (CJUAQ) cuenta con un área de $1.4 \mathrm{~km}^{2}$, de los cuales el $50 \%$ es selva baja caducifolia perturbada del cual se cuenta con un listado florístico de más de 120 especies (comm. pers. Martínez y Díaz de Salas 2012), el CJUAQ está rodeado por vías de comunicación importantes y se localiza en un área de contraste social ya que al norte colinda con una de las áreas residenciales con mayor plusvalía de la ciudad mientras que al este, se encuentra un área rural en desarrollo (comunidad de Rancho Largo).

Durante los meses de enero a mayo de 2010, se realizaron cinco periodos de trampeo de tres noches durante la primera semana de cada mes, se utilizaron 12 trampas tomahawk modelo 109.5 (107 x 31 x 31cm) (Tomahawk Live Trap Co.), separadas 50 m entre sí según lo permitiera el terreno y las obras de construcción; se utilizó como carnada una mezcla de sardina, alimento para gatos, cebolla y plátano; las trampas fueron activadas a partir del anochecer y fueron revisadas e inactivadas durante las primeras horas de la mañana; en caso de haber capturado algún animal, se transportó al laboratorio de Zoología para su manejo. Para la contención química, se utilizó Tiletamina, la dosis dependió del peso del animal $(10 \mathrm{mg} / \mathrm{kg})$ el cual se determinó por observación directa y se le administró intramuscularmente en los cuartos traseros. Una vez que la dosis hizo efecto, el animal fue colocado en posición recumbente y se procedió a la revisión de su estado físico (temperatura corporal, tasa de respiración y cardiaca) y a la toma de datos merísticos (longitudes del cuerpo, cola, oreja y pata derechas), peso, sexo y estimación de la edad por medio del esmalte dental (coloración de la dentadura y retraimiento de las encías).

Una vez que los animales se recuperaron de la anestesia, fueron liberados en la misma área donde fueron capturados. La captura y manejo de los animales se realizó siguiendo las consideraciones bioéticas reglamentarias sugeridas por The American Society of Mammalogists (Sikes et al. 2011).

Durante la etapa de recumbencia, el pelaje de los marsupiales fue revisado a contrapelo manualmente, los ectoparásitos fueron colectados con ayuda de pinzas y se fijaron con alcohol al $70 \%$ en un frasco con su etiqueta correspondiente por hospedero. Para el proceso de identificación, los parásitos fueron deshidratados inicialmente en alcohol etílico absoluto y posteriormente transparentados en concentraciones sucesivas de ácido láctico (25 al 100\%) hasta su montaje permanente con bálsamo de Canadá, la identificación se llevó a cabo por medio de literatura especializada (Acosta y Morrone 2003 y Durden y Hinkle 2009). 
Resultados

Durante este estudio, se capturaron 26 tlacuaches, 14 machos, 12 hembras; de los cuales el $50 \%$ del total de la muestra $(n=13)$ presentó ectoparásitos de las especies Ctenocephalides felis y Euhoplopsyllus glacialis affinnis de la familia Pulicidae y a Polygenis martinezbaezi y Polygenis sp. de la familia Rhoplopsyllidae, con un rango de infestación de 1 a 3 especies, siendo Ctenocephalides felis y Polygenis martinezbaezi las que presentaron la mayor prevalencia, 34.61 y $26.92 \%$ respectivamente, para toda la muestra. Con respecto a cada una de las especies de parásitos, se observa una mayor prevalencia en los machos que en las hembras, C. felis se registró en cinco de los 14 machos $(35.71 \%)$ a diferencia de las hembras, en donde fue registrada en cuatro de las 12 hembras (33.3\%), para P. martinezbaezi los machos están presentes un $28.57 \%$ ( $n$ $=4)$ mientras que las hembras en un $25 \%(n=3)$. Polygenis sp. fue registrada en dos machos (14.28\%), desafortunadamente el proceso de montaje dañó a los ejemplares y no fue posible lograr la identificación a nivel de especie; Euhoplopsyllus glacialis affinnis se encontró en un macho (7.14\%). Las preparaciones de las cuatro especies de pulgas fueron donadas a la Colección de Siphonaptera del Museo de Zoología de la Facultad de Ciencias Universidad Nacional Autónoma de México con el número de registros sucesivo SIPHO-08816 al SIPHO-08819.

La presencia de dos de las cuatro especies de pulgas (Euhoplopsyllus glacialis affinnis y Polygenis martinezbaezi) en Didelphis virginiana son nuevos registro para este marsupial en México, aunque estas especies de pulgas han sido registradas previamente en esta especie de hospedero en estudios realizados en Estados Unidos (Mohr y Morlan 1958; Whitaker et al. 1977 y Hopkins 1980) y en otras especies de marsupiales como Tlacuatzin canescens (Whitaker y Morales-Malacara 2005 y Guzmán-Cornejo et al. 2012). Especies de los géneros Ctenocephalides y Polygenis han sido registradas previamente en $D$. virginiania, Eckerlin (2011) identificó a C. canis como una de las pulgas más frecuentes en este marsupial en el estado de Virginia, Estados Unidos. Por otro lado, Pung et al. (1994) encontraron a Polygenis gwyni (C. fox) sobre el mismo marsupial en el estado de Georgia, mientras que un estudio en São Paulo, Brasil, fue registrada sobre Didelphis aurita y D. albiventris (Horta et al. 2007). Para México, la pulga Polygenis martinezbaezi fue registrada sobre Didelphis marsupialis en el estado de Michoacán, mientras que el género Euhoplopsyllus no se había asociado a D. virginiana, sin embargo, se le ha encontrado en ardillas (Otospermophilus variegatus y Xerospermophilus spilosoma), conejos del género Sylvilagus y roedores de las especies Neotoma albigula, Peromyscus boylii y Cynomys gunnisoni según lo reportado por Ford et al. (2004). De los huéspedes mencionados, algunos de estos cohabitan con los tlacuaches en la zona de estudio como es el caso de $O$. variegatus o de Sylvilagus floridanus. La presencia de estas especies de pulgas en $D$. virginiana puede ser una consecuencia del uso de madrigueras de otros animales, dicha actividad se considera como una estrategia de protección contra depredadores potenciales, principalmente de parte de las hembras con crías (McManus 1974) así como a la presencia de perros ferales en las cercanías del campus, con los cuales los tlacuaches pudieran Ilegar a tener contacto. 
Es importante hacer mención que el conocimiento de los ectoparásitos asociados con $D$. virginiana puede permitir reconocer el potencial de riesgo para la salud que representan las pulgas como vectores de microorganismos causantes de enfermedades tanto en fauna silvestre como en el ser humano; en el caso del hombre, dichas enfermedades han tenido un efecto importante a lo largo de la historia de nuestra civilización, una situación que ha quedado en el olvido debido a las mejoras en las condiciones de vida actuales que limitan la aparición de brotes epidemiológicos importantes, sin embargo, pueden regresar si se presentara inestabilidad de algún tipo, siendo ejemplos claros la peste y el tifus murino ocasionados por las bacterias Yersinia pestis y Rickettsia typhi respectivamente (Durden y Hinkle 2009), así como parásitos intestinales como es el céstodo Dipylidium caninum, que parasita perros, gatos y al hombre y del cual, las pulgas juegan un papel como hospedero intermediario del ciclo de vida de éste (Ford et al. 2004 y Acosta 2013).

Sin bien no se ha encontrado que $D$. virginianus sea reservorio de la peste, su actividad peridoméstica en viviendas periféricas de manchas urbanas y su probable interacción con roedores, pueden ocasionar la rápida diseminación de las pulgas hacia mascotas que interactúan con las personas, ya que se ha comprobado que al menos 125 especies de pulgas pueden transmitir $Y$. pestis, algunas de las cuales pertenecen a los géneros Ctenocephalides y Euhoplopsyllus (Barden y Maupin 1982 y Durden y Hinkle 2009) colectadas en esta investigación.

En el caso del tifus murino, sí se ha encontrado a $D$. virginianus como reservorio de la bacteria Rickettsia typhi (Horta et al. 2007 y Durden y Hinkle 2009), sin embargo los vectores de la bacteria son diferentes a los que aquí se encontraron asociados a este mamífero, no obstante como se indica anteriormente, la interacción con mascotas y con perros y gatos ferales, hacen que estas enfermedades sean un problema latente que se ha descuidado en los últimos años, por lo que, en caso de que dicha bacteria estuviera presente en la población de los tlacuaches del CJUAQ, la cercanía de la zona residencial y de la comunidad rural pueden significar un riesgo potencial epidemiológico para la transmisión de esta bacteria.

Este estudio, si bien inicialmente fue de carácter didáctico, arrojó información importante sobre la riqueza de especies de pulgas en tlacuaches que habitan ambientes en un proceso de transformación antropogénica y el efecto potencial de estos organismos para la salud de los seres humanos y de la fauna silvestre que se encuentran asociados a los mismos.

\section{Agradecimientos}

La primera autora desea agradecer al responsable del laboratorio de Zoología del Cuerpo Académico de Ecología y Diversidad Faunística (CALG, 2009-2012) por las facilidades otorgadas para la contención y manejo de los marsupiales capturados así como a la generación 2007 de la Licenciatura en Biología de la Universidad Autónoma de Querétaro por su entusiasmo y colaboración en la realización del estudio. 
Acosta, R., y J. J. Morrone. 2003. Clave ilustrada para la identificación de los taxones supraespecíficos de Siphonaptera de México. Acta Zoológica Mexicana (n. s.) 89:39-53.

Acosta-Gutiérrez, R. 2013. Biodiversidad de Siphonaptera en México. Revista Mexicana de Biodiversidad 84:1-12. DOI: 10.7550/rmb.35267.

Barnes, A. M., y G. O. Maupin. 1982. Observations on the biting of humans by Euhoplopsyllus glacialis affinis (Siphonaptera: Pulicidae) and a review of its plaguetransmission potential. Journal of Medicine Entomology 19:748-749.

Buskirk, S. W. 1999. Mesocarnivores of Yellowstone. Pp. 165-187 in Carnivores in ecosystems. The Yellowstone experience (T. W. Clark; A. P. Curlee; S. C. Minta y P. V. Kareiva eds.). Yale University Press, EE.UU.

Ceballos, G., P. Rodríguez, y R. A. Medellín. 1998. Assessing conservation priorities in megadiverse Mexico: mammalian diversity, endemicity, and endangerment. Ecological Applications 8:8-17.

Davies, K. F., C. Gascon, y C. R. Margules. 2001. Habitat Fragmentation. Pp 81-97 in Conservation Biology: Research priorities for the next decade. (Soulé M. E., y G. H. Orians eds.). Island Press. Washington, EE.UU.

Durden L. A., y N.C. Hinkle. 2009. Fleas (Siphonaptera). Pp. 110-130 in Medical and Veterinary Entomology (Mullen. G. R., y L, A. Durden, eds.). Second Edition. Elsevier. San Diego, EE.UU.

ECKerlin, R. P. 2011. What kind of flea does your dog have? Banisteria 37:42-43.

Ford Paulette l., R. A. Fagerlund, D. W. Duszynski, y P. J. Polechla. 2004. Fleas and Lice of Mammals in New Mexico. United States Department of Agriculture. General Technical Report RMRS-GTR-123. Washington, EE.UU.

Forman, R. T. T. y M. Godron. 1986. Landscape ecology. John Wiley \& Sons. New York, EE.UU.

García-Prieto, L., J. Falcón-Díaz, y C. Guzmán-Cornejo. 2012. Helminth parasites of wild Mexican mammals: lists of species, hosts and geographical distribution. Zootaxa 3290:1-92.

Gannon, W. L., R. S. Sikes y The Animal Care and Use Committee of The American Society of Mammalogists. 2011. Guidelines of the American Society of Mammalogists for the use of wild mammals in research. Journal of Mammalogy 92:235-253.

Gehring, T. M., y R. K. Swihart. 2003. Body size, niche breadth, and ecologically scaled responses to habitat fragmentation: mammalian predator in an agricultural landscape. Biological Conservation 109:283-295.

Guzmán-Cornejo, C., L. García-Prieto, R. Acosta-Gutiérrez, J. Falcón-Díaz, y L. LeónPaniagua. 2012. Metazoarios parásitos de Tlacuatlzin canescens y Marmosa mexicana (Mammalia: Didelphimorphia) de México. Revista Mexicana de Biodiversidad 83:557-561.

HARRISON, R. L. 1997. A comparison of gray fox ecology between residential and undeveloped rural landscapes. Journal of Wildlife Management 61:112-122. 
Hernández-Camacho, N., y C. A. López-González. 2009. ¿Hay o no hay? los mamíferos de Santiago de Querétaro. Universidad Autónoma de Querétaro. Nuevos Tiempos 2:3-6.

Hopkins, D. 1980. Ectoparasites of the Virginia opossum (Didelphis virginiana) in an urban environment. Northwest Science 54:199-201.

Horta-Maurício c., M. B. Labruna, A. Pinter, P. M. Linardi, y T. Schumaker. 2007. Rickettsia infection in five areas of the state of São Paolo, Brazil. Memórias do Instituto Oswaldo Cruz 102:793-801.

Mcmanus, J. J. 1974. Didelphis virginiana. Mammalian species 40:1-6.

Mohr, C. O., y H. B. Morlan. 1958. The nature of parasitism of the opossum by fleas in Southwestern Georgia. The Journal of Parasitology 45:233-237.

Morrone, J. J., y A. Gutiérrez. 2005. Do fleas (Insecta: Siphonaptera) parallel their mammal host diversification in the Mexican transition zone? Journal of Biogeography 32:1315-1325.

Pung, O. J., L. A. Durden, C. W. Banks, y D. N. Jones. 1994. Ectoparasites of opossums and raccoons in Southeastern Georgia. Journal of Medical Entomology 31:915919.

Sunquist, M., y F. Sunquist. 2001. Changing landscapes: consequences for carnivores. Pp. 399-418 in Conservation Biology 5. Carnivore Conservation. Cambridge University Press. The Zoological Society of London. London, Reino Unido.

Whitaker JR., J. O., G. S. Jones, y R. J. Goff. 1977. Ectoparasites and food habits of the Opossum, Didelphis virginiana, in Indiana. Proceedings Indiana Academy of Science 86:501-507.

Whitaker JR., J. O., y J. B. Morales-Malacara. 2005. Ectoparasites and other associates (Ectodytes) of mammals of Mexico in Contribuciones mastozoológicas en homenaje a Bernardo Villa (Sánchez-Cordero, V., y R. Medellín eds.). Instituto de Biología, Universidad Nacional Autónoma de México, Comisión Nacional para el Uso y Conocimiento de la Biodiversidad. Ciudad de México, México.

Whitaker JR., J. O., B. L. Walters, L. K. Castor, C. M. Ritzi, y N. Wilson. 2007. Host and distribution lists of mites (Acari), parasitic and phoretic, in the hair or on the skin of north American wild mammals north of Mexico: records since 1974. Faculty Publications from the Harold W. Manter Laboratory of Parasitology. Paper 1:1-174.

Sometido: 20 de enero de 2014

Revisado: 21 de marzo de 2014

Aceptado: 1 de abril de 2014

Editor asociado: Juan Pablo Gallo

Diseño gráfico editorial: Gerardo Hernández 
